\title{
The Intriguing Nature of the Cataclysmic Variable SS Cygni
}

\author{
F. Giovannelli, L. Sabau-Graziati
}

\begin{abstract}
The classification of SS Cyg as a dwarf nova (DN), a subclass of the non-magnetic (NM) cataclysmic variable (CV) has been considered by most of the community as well established because of a paper appeared in Nature (Bath \& van Paradijs, 1983), which was a bandwagon for all the papers discussing SS Cyg behaviour both from experimental and theoretical points of view. This classification has been widely accepted until nowadays, in spite of the many arguments and circumstantial proofs about its possible intermediate polar nature, as claimed by Franco Giovannelli's group for more than 25 years.

The goal of this paper is to present an objective discussion of the problems connected with the controversial nature of SS Cyg, using all the different interpretations of its multifrequency data in order to demonstrate beyond doubt its intermediate polar nature.
\end{abstract}

Keywords: cataclysmic variables, dwarf novae, intermediate polars, optical, spectroscopy, photometry, sub-mm, IR, radio, UV, X-rays, individual: SS Cyg $\equiv \mathrm{BD}+42^{\circ} 4189 \mathrm{a} \equiv \mathrm{AAVSO} 2138+43 \equiv 3 \mathrm{~A} 2140+433 \equiv 1 \mathrm{H} 2140+433 \equiv$ INTEGRAL1 $121 \equiv$ 1RXS J214242.6+433506 $\equiv$ EUVE J2142+43.6 $\equiv$ SWIFT J2142.7+4337.

\section{Introduction}

Historically, the classification of $\mathrm{CVs}$ was based on the optical outburst properties, by which one may distinguish four groups of CVs: (i) classical novae; (ii) recurrent novae; (iii) dwarf novae; (iv) nova-like objects (e.g., Giovannelli \& Martinez-Pais, 1991 and references therein; Ritter, 1992; Giovannelli, 2008). This classification, however, is neither self-consistent nor adequate, and it is much better to consider primarily the observed accretion behaviour (Smak, 1985). One obvious advantage of such an approach is connected with the time scales of various accretion phenomena, which are sufficiently short to avoid any major observational bias. The mass accretion rates in CVs usually range from $10^{-11}$ to $10^{-8} \mathrm{M}_{\odot} \mathrm{yr}^{-1}$ (Patterson, 1984); the time scales are from tens of seconds (oscillations in dwarf novae at outbursts) to years (super-outbursts of SU UMa stars or long term variations in VY Scl stars). However, in the class of nova-like objects there are two sub-classes: DQ Her stars and AM Her stars. In these sub-classes of CVs, white dwarfs possess magnetic fields with intensity high enough to dominate the accretion disk and all the phenomena related to it. These classes of magnetic CVs, whose names come from the prototypes DQ Her and AM Her, later took the names of Intermediate Polars and Polars, respectively. A short history of their discovery was discussed by Warner (1995). Fundamental papers about these sub-classes are those by Patterson (1994), Warner (1996a,b). The class of IPs has been split into two subclasses with relatively a large magnetic field anf with a relatively weak magnetic field (Norton et al., 1999). One example of a system belonging to the latter subclass is DO Dra (previously registered as YY Dra) (Andronov et al., 2008).

Depending on the magnetic field intensity at the white dwarf, the accretion of matter from the secondary star onto the primary can occur either via an accretion disc (in the so-called Non-Magnetic CVs: NMCVs) or via channelling through the magnetic poles (in the case of Polars: PCVs), or in an intermediate way (in the case of Intermediate Polars: IPCVs).

SS Cyg is the most observed and most intriguing CV. For reviews, see the papers by Giovannelli \& Martinez-Pais (1991), Giovannelli (1996), Giovannelli \& Sabau-Graziati (1998). The most extensive review about SS Cyg before the advent of the space era is that by Zuckerman (1961). The light curves of SS Cyg have been produced continuously by the AAVSO observations since 1896 (Mattei, et al., 1985; Mattei, Waagen \& Foster, 1991, 1996; Mattei, Menali \& Waagen, 2002; AAVSO web page (http://www.aavso.org/)).

The optical outbursts of SS Cyg are not always the same. Howarth (1978) discussed three possible kind of outbursts, long, short and anomalous with average periodicity of 50.21 days. Giovannelli et al. (1985), Lombardi, Giovannelli \& Gaudenzi (1987), Gaudenzi et al. (1990; 2011) and Giovannelli \& Martinez-Pais (1991) discussed outbursts that originate different optical, UV and X-ray behaviour of the system.

On the basis of its optical light curves, SS Cyg was classified as a dwarf nova (Bath \& van Paradijs, 1983), with white dwarf mass equal to 
Chandrasekhar's limit (Patterson, 1981). However, we will discuss its intermediate polar nature, analyzing its multifrequency behaviour and different interpretations of the data from the literature. Moreover, on the basis of more realistic values for its orbital parameters, we will try to reconcile all experimental multifrequency data with the magnetic nature of SS Cyg.

\section{On the controversial nature of SS Cyg}

With the historical classification of CVs based on the optical outburst properties, SS Cyg $\left(\alpha_{2000}=\right.$ $21^{h} 42^{m} 48^{s} .2 ; \delta_{2000}=+43^{\circ} 35^{\prime} 09^{\prime \prime} .88$ with the galactic coordinates $\left.l_{2000}=090.5592, b_{2000}=-07.1106\right)$, whose distance is $166.2 \pm 12.7 \mathrm{pc}$ (Harrison et al., 1999), is the brightest of the dwarf novae. Its optical magnitude ranges from $\sim 12$ to $\sim 8.5$ during quiescent and outburst phases, respectively.

Because of these characteristics, it is the most observed CV, not only in the optical wavelength region, where measurements are available from the end of the 19th century to the present, but also in other wavelength regions.

SS Cyg shows oscillations of $\sim 10 \mathrm{~s}$ in both the optical and the $\mathrm{X}$-ray ranges, orbital modulations $\left(\mathrm{P}_{\text {orb }} \simeq 6.6 \mathrm{~h}\right)$ of the intensities of Balmer and UV emission lines and of the continuum, and almost periodic outbursts $\left(\mathrm{P}_{\text {outb }} \sim 50\right.$ days, Howarth, 1978). All these characteristics, together with relatively high luminosity both in outburst and in quiescence, render SS Cyg the most appropriate laboratory for studying the physical processes occurring in dwarf novae and in CVs in general.

The orbital parameters of the binary system were derived by Giovannelli et al. (1983) with the use of theoretical and experimental constraints from measurements obtained in different energy regions. They are $i=40^{\circ}{ }_{-2}^{+1}, \mathrm{M}_{1}=0.97_{-0.05}^{+0.14} \mathrm{M}_{\odot}$, $\mathrm{M}_{2}=0.56_{-0.03}^{+0.08} \mathrm{M}_{\odot}, \mathrm{R}_{2}=0.68_{-0.01}^{+0.03} \mathrm{R}_{\odot}, \mathrm{R}_{\text {od }}=$ $2.9 \times 10^{10} \mathrm{~cm}, \mathrm{R}_{\mathrm{id}}=3.6 \times 10^{9} \mathrm{~cm}$, where 1 and 2 refers to the primary and secondary star, respectively. $R_{\text {od }}$ and $R_{\text {id }}$ are the outer and inner accretion disk radius. These parameters have been confirmed by direct measurements of radial velocities (Martinez-Pais et al., 1994). Martinez-Pais et al. also determined that the optical companion of SS Cyg system is a K2-K3 late-type star.

The mass of the white dwarf of the binary system SS Cyg was considered for long time as high as Chandrasekhar's limit - because of a paper that appeared in the Astrophysical Journal (Patterson, 1981) - a value completely unuseful for any sort of serious interpretation of the many multifrequency experimental data and for modeling.
In a study of the matter flow structure in SS Cyg using the Doppler tomography technique, Bisikalo et al. $(2008)$ found that $R_{\mathrm{id}}=(2.6-3.3) \times 10^{9} \mathrm{~cm}$, which is another important confirmation of the goodness of Giovannelli et al.'s parameters.

Despite the enormous amount of multifrequency experimental data spread over many years, the morphology and the nature of SS Cyg are still unsettled questions. Indeed, SS Cyg was classified as a non-magnetic CV (NMCV) by Bath \& van Paradijs (1983). Ricketts, King \& Raine (1979) explained the X-ray emission from SS Cyg as owing to the radial inflow of matter onto a magnetized white dwarf $\left(B \sim 10^{6} \mathrm{G}\right)$ from a disrupted accretion disk. Fabbiano et al. (1981), using coordinated optical-UV and X-ray measurements of SS Cyg, noted that its behavior is not compatible with a viscous disk model and confirmed the magnetic nature of the white dwarf with $B \leq 1.9 \times 10^{6} \mathrm{G}$. SS Cyg at quiescence is quite similar to AM Her and its behaviour is consistent with a picture of polar magnetic accretion. Further multifrequency data of SS Cygni showed incompatibility of its behavior with that of NMCV, and strongly favored its classification as an intermediate polar (see, e.g. Giovannelli et al., 1985; Giovannelli \& Martinez-Pais, 1991; Kjurkchieva, Marchev \& Ogłoza, 1999; Marchev, Kjurkchieva \& Ogłoza, 1999; Gaudenzi et al., 2002; Long et al., 2005; Schreiber, Hameury \& Lasota, 2003; Schreiber, \& Lasota, 2007).

Moreover, in SS Cyg, $\mathrm{L}_{\text {hard }-\mathrm{X}}<\mathrm{L}_{\mathrm{UV}+\text { soft }-\mathrm{X}}$. This is compatible with thermonuclear burning onto the WD surface. Thermonuclear burning was first suggested by Igor Mitrofanov (1978). Gaudenzi et al. (2002) found that thermonuclear burning can occur in $\sim 24 \%$ of the WD surface. Kording et al. (2008) detected a radio jet from SS Cyg. The hardness intensity diagram shows an analogy between X-ray binaries (XRBs) and SS Cyg. This result supports the presence of a rather strong magnetic field at the surface of the white dwarf. Upper limits to linear and circular polarizations have been found as $3.2 \pm 2.7 \%$ and $-3.2 \pm 2.7 \%$, respectively.

INTEGRAL/IBIS and SWIFT/XRT observations have shown that a conspicuous number of CVs have a strong hard X-ray emission (Landi et al., 2009; Scaringi et al., 2010). In their published sample of $22 \mathrm{CVs}, 21$ are classified as magnetic CVs (MCVs) (intermediate polar: IP) and only one (SS Cyg) as NMCV, meanwhile all its characteristics are practically equal to those of the other 21 objects. This is one more strong circumstantial proof in favor of the magnetic nature of SS Cyg. Scaringi et al. (2010) reported the detection of one more IP: AO Psc, which is added to the former sample. The experimental evidence that SS Cyg emits in the hard X-ray energy range is, in our opinion, conclusive evidence about its magnetic nature. 
However, there are many papers in the literature that seem to contradict the intermediate polar nature of SS Cyg. Indeed, Gnedin et al. (1995) found from observations of intrinsic circular polarization in SS Cyg performed in the wings of the Balmer hydrogen lines that the true value of the magnetic field probably lies in the range $0.03<\mathrm{B}<0.3 \mathrm{MG}$.

Using Extreme Ultraviolet Explorer satellite observations, Mauche (1996) detected quasi-periodic oscillations (QPOs) from SS Cyg with a period in the range $7.19-9.3 \mathrm{~s}$. This variation correlates with the extreme ultra-violet (EUV) flux as $P \propto I_{\mathrm{EUV}}^{-0.094}$. With a magnetospheric model to reproduce this variation, he found that a high-order, multipole field is required, and that the field strength at the surface of the white dwarf is $0.1<\mathrm{B}<1 \mathrm{MG}$. This field strength is at the lower extreme of those measured or inferred for bona fide magnetic cataclysmic variables. However, they do not exclude the possibility that at an outburst the accretion of matter could occur onto the magnetic poles of the white dwarf. Giovannelli (1981) found QPOs from SS Cyg — during a rise up to a maximum of one long optical outburst — with periods in the range 8.96-9.91 s that show the inverse relationship between outburst luminosity and oscillation period as general properties of CVs (e.g. Nather \& Robinson, 1974; Nevo \& Sadeh, 1978). The amplitude of the optical oscillations has a maximum at the maximum of the outburst. Then the white dwarf is heated directly by the surrounding luminous material, as by accretion, as suggested by Hildebrand et al. (1980) for AH Her. The variation of the rapid oscillation periods in SS Cyg has the same trend with respect to $\mathrm{AH}$ Her. The temperature is minimum at the peak of the outburst. A possible explanation could be that very close to the optical maximum the inner part of the disk moves closer to the white dwarf surface and it becomes denser and renders cooling possible. This optical behaviour is in agreement with the X-ray behaviour of SS Cyg. Indeed, at the optical maximum, the hard X-ray emission is lower than during quiescence, and all the energy in the X-ray region is emitted below $2 \mathrm{keV}$ (Ricketts, King \& Raine, 1979).

With regard to the question of the possible magnetic nature of SS Cyg, Mauche et al. (1997) discussed the case of the UV line ratios of CVs, which seem to be almost independent of the nature (magnetic or not) of CVs. Okada, Nakamura \& Ishida (2008) found from CHANDRA HETG observations in SS Cyg that the spectrum in quiescence is dominated by $\mathrm{H}$-like $\mathrm{K}_{\alpha}$ lines, and is dominated in outburst by He-like lines, which are as intense as H-like lines. The broad line widths and line profiles indicate that the line-emitting plasma is associated with the Keplerian disk. In quiescence the lines are narrower and are emitted from an ionizing plasma at the en- trance of the boundary layer. Ishida et al. (2009) found from SUZAKU observations of SS Cyg that the plasma temperature in quiescence is $20.4 \mathrm{keV}$ and in outburst $6.0 \mathrm{keV}$. The $6.4 \mathrm{keV}$ line is resolved in narrow and broad components, which indicates that both the white dwarf and the accretion disk contribute to the reflection. The standard optically thin boundary layer is the most plausible picture of the plasma configuration in quiescence. The reflection in outburst originates from the accretion disk and an equatorial accretion belt. The broad $6,4 \mathrm{keV}$ line suggests that the optically thin thermal plasma is distributed on the accretion disk, in a manner similar to that of a solar corona.

Long et al. (2005) found by fitting the doublepeaked line profile in SS Cyg that the FUV lineforming region is concentrated closer to the white dwarf than the region that forms the optical lines. Their study provides no evidence of a hole in the inner disk. However, they cannot fit SS Cyg data by a simple model as white dwarf plus accretion disk.

The SS Cyg system is also important as laboratory for the study of circumstellar dust in CVs. Indeed, Jameson et al. (1987) detected IR emission from SS Cyg in outburst in IRAS Bands I and II $(11.8 \mu \mathrm{m}$ and $24.4 \mu \mathrm{m})$. The most likely origin of the IR emission is circumstellar dust heated by the enhanced UV flux during outburst. Dubus et al. (2004) performed optical and mid-IR observations of several CVs including SS Cyg in quiescence. For SS Cyg the measurements at $11.8 \mu \mathrm{m}$ are consistent with the upper limits obtained by Jameson et al. (1987) when the source was not yet in full outburst. The observed variability in the mid-IR flux on short time scales is hardly reconcilable with intrinsic or reprocessed emission from circumbinary disk material, while on the contrary a free-free emission from a wind should be reconcilable. If any sizeable circum-binary disk is present in the system, it must be self-shadowed or perhaps dust-free, with the peak thermal emission shifted to far-IR wavelengths.

Gaudenzi et al. (2011) discussed the reasons for the variable reddening in SS Cyg and demonstrated that this reddening consists of two components: the first is interstellar in origin, and the second (intrinsic to the system itself) is variable and changes during the evolution of a quiescent phase. Moreover, an orbital modulation also exists. The physical and chemical parameters of the system are consistent with the possibility of the formation of fullerenes.

The SPITZER space telescope detected an excess (3-8) $\mu \mathrm{m}$ emission from Magnetic CVs, due to dust (Howell et al., 2006; Brinkworth et al., 2007). This is a strong push for observing SS Cyg carefully with SPITZER. However, a weak IR excess was discovered in SS Cyg looking at the SPITZER data, but no conclusions were given since the data at different 
Table 1: Comparison of the characteristic parameters of EI UMa - a well established IP (Reimer et al., 2008) — and SS Cyg (Giovannelli et al., 1983; Lombardi et al., 1987; Gaudenzi et al., 1990, 2002; Giovannelli \& Martinez-Pais, 1991; Schreiber \& Gänsicke, 2002)

\begin{tabular}{|c|c|}
\hline EI UMa & SS Cyg \\
\hline $\mathrm{P}_{\text {orb }}=6.434 \mathrm{~h}$ & $\mathrm{P}_{\text {orb }}=6.603 \mathrm{~h}$ \\
\hline $\mathrm{P}_{\mathrm{opt}}=745 \mathrm{~s}\left(\sim \mathrm{P}_{\mathrm{XMM}}\right)$ & $\mathrm{P}_{\mathrm{opt}}=745 \mathrm{~s}\left(\sim \mathrm{P}_{\mathrm{XMM} \mathrm{UV}}\right)$ \\
\hline $0.81 \mathrm{M}_{\odot}<\mathrm{M}_{\mathrm{WD}}<1.2 \mathrm{M}_{\odot}$ & $\mathrm{M}_{\mathrm{WD}}=0.97 \mathrm{M}_{\odot}$ \\
\hline $\mathrm{R}_{\mathrm{WD}}=7 \times 10^{8} \mathrm{~cm}$ & $\mathrm{R}_{\mathrm{WD}}=5 \times 10^{8} \mathrm{~cm}$ \\
\hline $\mathrm{M}_{\mathrm{R}}=0.81 \mathrm{M}_{\odot}$ & $\mathrm{M}_{\mathrm{R}}=0.56 \mathrm{M}_{\odot}$ \\
\hline $\mathrm{R}_{\mathrm{R}}=0.76 \mathrm{R}_{\odot}$ & $\mathrm{R}_{\mathrm{R}}=0.68 \mathrm{R}_{\odot}$ \\
\hline $\mathrm{L}_{\mathrm{X}} \sim 10 \times 10^{32} \mathrm{erg} \mathrm{s}^{-1}$ & $\mathrm{~L}_{\mathrm{X}} \sim(6.6-9.8) \times 10^{32} \mathrm{erg} \mathrm{s}^{-1}$ \\
\hline$\dot{\mathrm{M}}=3.6 \times 10^{17} \mathrm{~g} \mathrm{~s}^{-1}$ & $\dot{\mathrm{M}} \simeq(1-4) \times 10^{17} \mathrm{~g} \mathrm{~s}^{-1}$ \\
\hline $\mathrm{M}_{V}=5.4$ & $\mathrm{M}_{V}=5.9$ \\
\hline $\mathrm{f}=\mathrm{Rd} / \mathrm{a}=0.2-0.3$ & $\mathrm{f}=\mathrm{Rd} / \mathrm{a}=0.2$ \\
\hline $\begin{array}{l}\text { UBV Orbital modulations of } \\
\text { continuum }\end{array}$ & $\begin{array}{l}\text { UBV \& UV Orbital modulations of } \\
\text { continuum \& emission lines EWs }\end{array}$ \\
\hline
\end{tabular}

wavelengths were not simultaneous (Harrison et al., 2010).

\section{The intermediate polar nature of SS Cyg}

In our opinion, there are several incontestable arguments in favour of the IP nature of SS Cyg, namely:

i) the strong analogy of SS Cyg with the well established IP EI UMa (Reimer et al., 2008). Table 1 shows the parameters of EI UMa and SS Cyg;

ii) in the diagram $\log L_{X}$ vs $\log \dot{M}$ for the IPs (Warner, 1996a), SS Cyg lies just in the place of IPs (Figure 1, left panel). The X-ray luminosity of SS Cyg $-\mathrm{L}_{X} \sim(6.6-9.8) \times 10^{32} \mathrm{erg} \mathrm{s}^{-1}-$ has been derived by the values of the distance of SS Cyg: 166 pc (Harrison et al., 1999) and its X-ray flux: $\mathrm{F}_{X} \sim(2-3) \times 10^{-10} \mathrm{erg} \mathrm{cm}^{-2} \mathrm{~s}^{-1}$ (Giovannelli \& Martinez-Pais, 1991; McGowan, Priedhorsky \& Trudolyubov, 2004). The average mass accretion rate is $\dot{\mathrm{M}} \sim 2 \times 10^{17} \mathrm{~g} \mathrm{~s}^{-1}$ (Gaudenzi et al., 1990, and the references therein; Schreiber \& Gänsicke, 2002);

iii) in the diagram $\log \dot{\mathrm{M}}$ vs $\log \mathrm{P}_{\text {orb }}$ (Warner, 1996a) SS Cyg lies just in the place of IPs (Fig. 1, right panel). The orbital period of SS Cyg is $\mathrm{P}_{\text {orb }}=6.6 \mathrm{~h}$ (Martinez-Pais et al. 1994);

iv) SS Cyg has been detected in the hard X-ray range, together with other tens of very well known IPs (Landi et al., 2009; Scaringi et al., 2010). Such an emission cannot be justified without the presence of an intense white dwarf magnetic field. Moreover, if SS Cyg should be a dwarf nova (non-magnetic CV) as reported in the table of detected CVs in the former papers - why are other dwarf novae not detected with the same instruments?
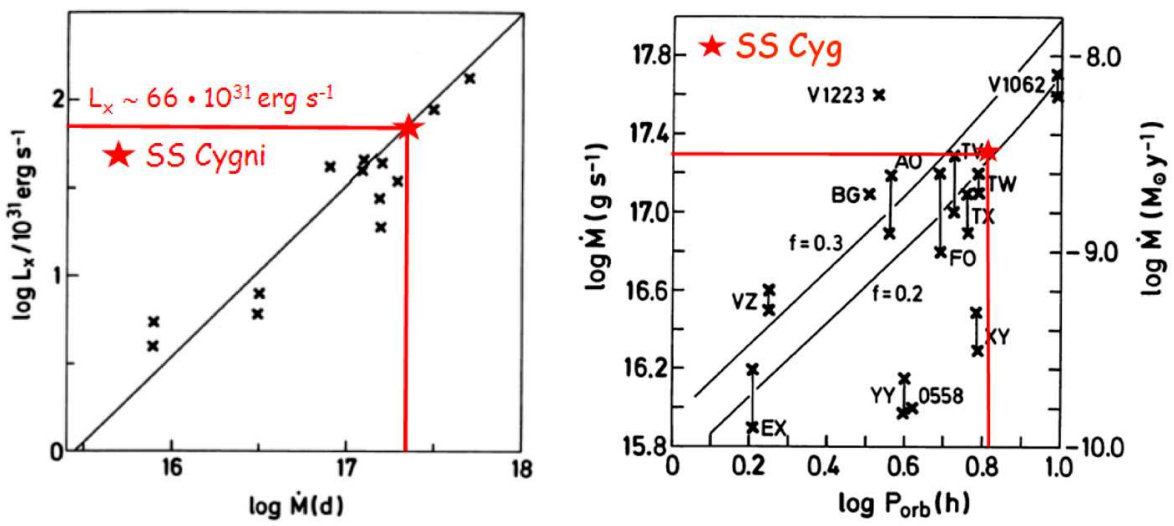

Fig. 1: Left panel: relationship between X-ray luminosity and mass accretion rate for IPs that are indicated with $\mathrm{x}$ (by courtesy of Warner, 1996a). Right panel: relationship between mass accretion rate and orbital period for IPs (by courtesy of Warner, 1996a). SS Cyg positions are indicated with a red $\star$ in both panels 

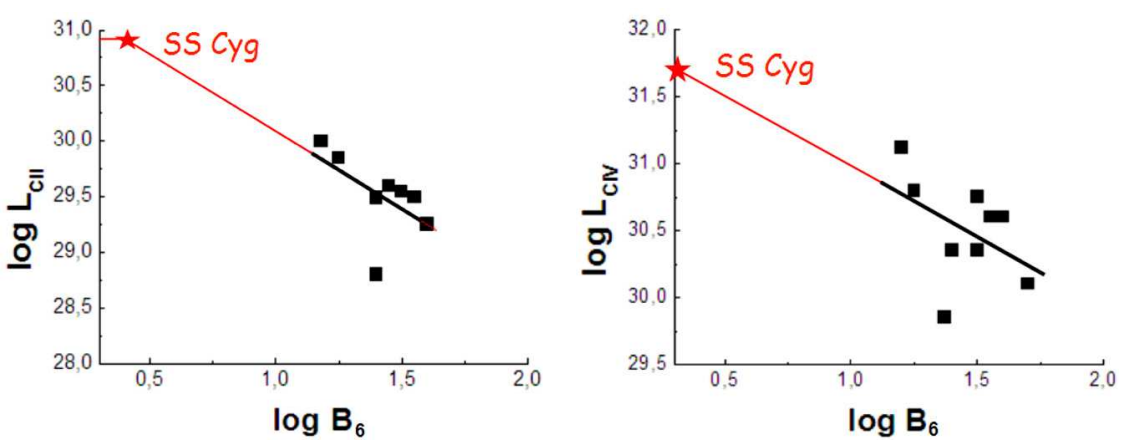

Fig. 2: Observed emission fluxes converted to luminosity for magnetic CVs (after Howell et al., 1999) are indicated with -: C II in the left panel, and C IV in the right panel. SS Cyg positions are indicated with a red $\star$. Luminosity expressed in erg $\mathrm{s}^{-1}$, and $\mathrm{B}$ in unit of $10^{6}$ gauss
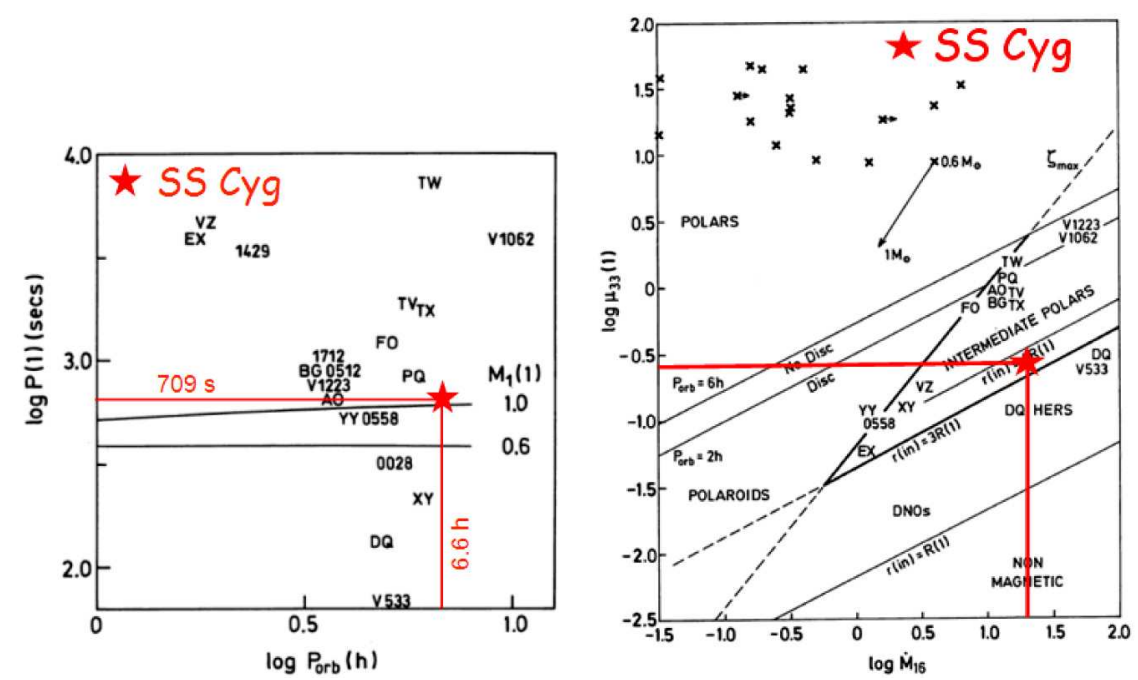

Fig. 3: Left panel: relationship between $\mathrm{P}_{\text {spin }}$ and $\mathrm{P}_{\text {orb }}$ for IPs (by courtesy of Warner, 1996a). Right panel: relationship between the magnetic moment of the white dwarfs in CVs and $\mathrm{M}_{\odot}$ (by courtesy of Warner, 1996a). Polars are indicated with $\mathrm{x}$, and IPs with their abbreviated titles. SS Cyg positions are indicated with a red $\star$. Magnetic moment $\mu$ is expressed in $\mathrm{G} \mathrm{cm}^{3}$, and $\mathrm{M}_{\odot}$ in unit of $10^{16} \mathrm{~g} \mathrm{~s}^{-1}$

Rudolf Gális et al. (2011, IBWS) when discussing the X-ray and optical activity of INTEGRAL CVs, explicitly mentioned SS Cyg as IP, making a comparison of its X-ray behaviour with those very similar of V 709 Cas, a well-established IP.

Then we can assume, beyond reasonable doubt, that SS Cyg is an IP. What is the intensity of its magnetic field? A relationship between the strength of high-state UV emission lines and the strength of the white dwarf magnetic field has been found by Howell et al. (1999).

From the fluxes of UV emission lines (Gaudenzi et al., 1986) we derived the luminosity of C II and C IV: $\mathrm{L}_{\mathrm{CII}} \sim 7.8 \times 10^{30} \mathrm{erg} \mathrm{s}^{-1}$, and $\mathrm{L}_{\mathrm{CIV}} \sim 6.2 \times$ $10^{31} \mathrm{erg} \mathrm{s}^{-1}$, respectively.

Using these values of luminosity and the extrapolation of the line best fitting the emission line luminosity of C II and C IV from Howell et al. (1999), the magnetic field intensity of SS Cyg is 2.5 and 2.0 MG, respectively as shown in Fig. 2, left and right panels, respectively.

Taking into account the errors in the fluxes of the considered UV lines, we can argue a reasonable value of the white dwarf magnetic field in SS Cyg as $\mathrm{B} \sim 2.2 \pm 1.0 \mathrm{MG}$.

Moreover,

$\mathrm{v})$ a periodicity at $12.18 \pm 0.01 \mathrm{~m}$ in $\mathrm{R}$ and $\mathrm{I}$ bands was detected in SS Cyg by Bartolini et al. (1985), probably the beat period between the spin period of the white dwarf and the orbital period of the system. If the rotation is direct with orbital motion $\mathrm{P}_{\text {spin }}=11.82 \pm 0.01 \mathrm{~m}$, corresponding to $709.2 \pm 0.6 \mathrm{~s}$. If the rotation is inverse $\mathrm{P}_{\text {spin }}=12.56 \pm 0.01 \mathrm{~m}$ $(753.6 \pm 0.6 \mathrm{~s})$. Tramontana (2007) found a periodicity of $12.175 \pm 0.539 \mathrm{~m}$ using 504 images obtained in the R band on October 26, 2006 with SS Cygni in outburst at the TACOR teaching telescope of La Sapienza University. Using 10 ks XMM-OM UV ob- 
servations of SS Cyg, Braga (2009) found a period of $709 \pm 1 \mathrm{~s}$ that should be the rotational period of the white dwarf, following the model of IPs developed by Warner (1986).

Using $\mathrm{P}_{\text {spin }}=709 \mathrm{~s}$ and the relationship $\mathrm{P}_{\text {spin }}$ vs $\mathrm{P}_{\text {orb }}$ (Warner, 1996a), SS Cyg lies in the zone of the IPs very close to the line of $1 \mathrm{M}_{\odot}$ (Figure 3, left panel). The mass of the SS Cyg white dwarf is $0.97_{-0.05}^{+0.14} \mathrm{M}_{\odot}$ (Giovannelli et al., 1983).

Finally, with $\mathrm{B} \approx 2 \mathrm{MG}$, and the radius of the white dwarf $\mathrm{R}_{\mathrm{wd}}=5 \times 10^{8} \mathrm{~cm}$ (Martinez-Pais et al., 1994 ), the magnetic moment is $\mu \approx 2.5 \times 10^{32} \mathrm{G} \mathrm{cm}^{3}$. Using this value of $\mu$ and the average value of $\dot{M}$, SS Cyg lies just in the IPs region, as shown in the right panel of Figure 3.

Therefore, we can definitively affirm that SS Cyg is an intermediate polar.

\section{Acknowledgement}

We are pleased to thank the organizers of the Karlovy Vary 8th INTEGRAL/BART Workshop for the invitation. One of us (FG) wishes to thank the LOC for logistical support.

This research has made use of NASA's Astrophysics Data System.

\section{References}

[1] Andronov, I. L., Chinarova, L. L., Han, W., Kim, Y., Yoon, J.-N.: $A \&$ \& $A, 2008,486,855$.

[2] Bath, G. T., van Paradijs, J.: Nature, 1983, 305, 33.

[3] Bartolini, C., et al.: Multifrequency Behaviour of Galactic Accreting Sources, F. Giovannelli (ed.) Edizioni Scientifiche SIDEREA, Roma : 1985 , p. 50 .

[4] Bisikalo, D. V., et al.: Astron. Rep., 2008, 52, No. 4, 318.

[5] Braga, V. F.: Thesis in Physics, Roma : 2009, University La Sapienza.

[6] Brinkworth, C. S., et al: 15th European Workshop on White Dwarfs, R. Napiwotzki, M. R. Burleigh (eds.) ASP Conf. Ser. 2007, 372, 333.

[7] Dubus, G. et al.: $M N R A S, 2004, \mathbf{3 4 9}, 869$.

[8] Fabbiano, G., et al.: ApJ, 1981, 243, 911.

[9] Galis, R., et al.: 2011, this workshop.

[10] Gaudenzi, S., Giovannelli, F., Lombardi, R., Claudi, R.: New Insights in Astrophysics. Eight
Years of UV Astronomy with IUE, $E S A-S P$, 1986, 263, 455.

[11] Gaudenzi, S., Giovannelli, F., Lombardi, R., Claudi, R.: AcA, 1990, 40, 105.

[12] Gaudenzi, S., et al.: Multifrequency Behaviour of High Energy Cosmic Sources, F. Giovannelli, L. Sabau-Graziati (eds.), Mem. S.A.It. 2002, 73, N. 1, 213.

[13] Gaudenzi, S., et al.: $A \mathscr{G} A, 2011, \mathbf{5 2 5}, 147$.

[14] Giovannelli, F.: SSR, 1981, 30, 213.

[15] Giovannelli, F.: Multifrequency Behaviour of High Energy Cosmic Sources, F. Giovannelli, L. Sabau-Graziati (eds.) Mem. S.A.It. 1996, 67, 401.

[16] Giovannelli, F.: ChJA \& A, 2008, 8 Suppl., 237.

[17] Giovannelli, F., Gaudenzi, S., Piccioni, A., Rossi, C.: AcA, 1983, 33, 319 .

[18] Giovannelli, F., et al.: Multifrequency Behaviour of Galactic Accreting Sources, F. Giovannelli (ed.) Edizioni Scientifiche SIDEREA, Roma : 1985, p. 37.

[19] Giovannelli, F., Martinez-Pais, I. G.: Space Sci. Rev., 1991, 56, 313.

[20] Giovannelli, F., Sabau-Graziati, L.: Ultraviolet Astrophysics Beyond the IUE Final Archive, W. Wamsteker, R. Gonzalez-Riestra (eds.) ESA SP, 1998, 413, 419 .

[21] Gnedin, Yu. N., et al.: Astr. Lett., 1995, 21, 132.

[22] Harrison, T. E., et al.: ApJL, 1999, 515, L93.

[23] Harrison, T. E., et al.: ApJ, 2010, 710, 325.

[24] Howarth, I. D.: J. Brit. Astron. Ass., 1978, 88, 458.

[25] Howell, S. B., et al.: AJ, 1999, 117, 1014.

[26] Howell, S. B., et al.: ApJL, 2006, 646, L65.

[27] Ishida, M., et al.: PASJ, 2009, 61, S77.

[28] Jameson, R. F., et al.: Observatory, 1987, 107, 72 .

[29] Kjurkchieva, D., Marchev, D., Ogłoza, W.: Ap \&6 $S S, 1999, \mathbf{2 6 2}, 53$.

[30] Körding, E., et al.: Science, 2008, 320, 1318.

[31] Landi, et al.: MNRAS, 2009, 392, 630.

[32] Lombardi, R., Giovannelli, F., Gaudenzi, S.: Ap \& SS, 1987, 130, 275. 
[33] Long, K. S., et al.: ApJ, 2005, 630, 511.

[34] Marchev, D., Kjurkchieva, D., Ogłoza, W.: AcA, 1999, 49, 585 .

[35] Martinez-Pais, I. G., Giovannelli, F., Rossi, C., Gaudenzi, S.: $A$ \& $A, 1994, \mathbf{2 9 1}, 455$.

[36] Mattei, J. A., Saladyga, M., Waagen, E. O.: SS Cygni Light Curves 1896-1985, AAVSO Monograph 1, Cambridge : MA, 1985.

[37] Mattei, J. A., Waagen, E. O., Foster, E. G.: SS Cygni Light Curves 1985-1990, AAVSO Monograph 1 Supplement 1, Cambridge : MA, 1991.

[38] Mattei, J. A., Waagen, E. O., Foster, E. G.: SS Cygni Light Curves 1991-1995, AAVSO Monograph 1 Supplement 2, Cambridge : MA, 1996.

[39] Mattei, J. A., Menoli, H. G., Waagen, E. O.: SS Cygni Light Curves 1996-2000, AAVSO Monograph 1 Supplement 3, Cambridge : MA, 2002.

[40] Mauche, C., et al.: ApJL, 1996, 463, L87.

[41] Mauche, C., et al.: ApJ, 1997, 477, 832.

[42] McGowan, K. E., Priedhorsky, W. C., Trudolyubov, S. P.: ApJ, 2004, 601, 1100.

[43] Mitrofanov, I. G.: Sov. Astron. Lett., 1978, 4, 119.

[44] Nather, R. E., Robinson, E. L.: ApJ, 1974, 190, 637.

[45] Nevo, I., Sadeh, D.: MNRAS, 1978, 182, 595.

[46] Norton, A. J., Beardmore, A. P., Allan, A., Hellier, C.: $A \& \&$ A, 1999, 347, 203.

[47] Ricketts, M. J., King, A. R., Raine, D. J.: $M N$ $R A S$, 1979, 186, 233.

[48] Ritter, H.: The Astronomy and Astrophysics Encyclopedia. Cambridge, UK : Cambridge University Press, 1992, p. 61.

[49] Okada, S., Nakamura, R., Ishida, M.: ApJ, 2008, 680, 695 .

[50] Patterson, J.: ApJS, 1981, 45, 517.
[51] Patterson, J.: ApJS, 1984, 54, 443.

[52] Patterson, J.: PASP, 1994, 106, 209.

[53] Reimer, T. W., et al.: ApJ, 2008, 678, 376.

[54] Scaringi, S., et al.: MNRAS, 2010, 401, 207.

[55] Schreiber, M. R., Gänsicke, B. T.: $A \& \mathcal{E} A, 2002$, 382, 124.

[56] Schreiber, M. R., Lasota, J.-P.: $A \& \xi A, 2007$, 473, 897.

[57] Schreiber, M. R., Hameury, J.-M., Lasota, J.-P.: $A$ \& $A, 2$ 2003, 410, 239.

[58] Smak, J.: Multifrequency Behaviour of Galactic Accreting Sources, F. Giovannelli (ed.) Edizioni Scientifiche SIDEREA, Roma : 1985, p. 17.

[59] Tramontana, V.: Thesis in Physics. Roma: University La Sapienza, 2007.

[60] Warner, B.: MNRAS, 1986, 219, 347.

[61] Warner, B.: Cape Workshop on Magnetic Cataclysmic Variables, D. A. H. Buckley, B. Warner (eds.) ASP Conf. Ser., 1995, 85, 3.

[62] Warner, B.: Ap \&S SS, 1996, 241, 263.

[63] Warner, B.: Cataclysmic Variable Stars, Cambridge University Press, 1996.

[64] Zuckerman, M.-C.: Ann. Astrophys., 1961, 6, 431.

Franco Giovannelli

INAF - Istituto di Astrofisica

Spaziale e Fisica Cosmica - Roma

Area di Ricerca di Roma-2

Via del Fosso del Cavaliere 100, I 00133 Roma, Italy

Lola Sabau-Graziati

INTA - Dpt de Programas Espaciales y Ciencias del Espacio

Ctra de Ajalvir Km 4 - E 28850 Torrejón de Ardóz, Spain 